\title{
Prediction of the Burden of Released Mental Patients
}

\author{
George Williams, Ph.D.* \\ Fu-tong Hsu, Ph.D. \\ Tsung-yi Lin, M.D.
}

ABSTRACT: The burden of the patient to his family and the community is a major factor influencing the success of community care of a psychiatric patient. The hospital staff should be able to forsee it and take it into consideration in planning the aftercare of the psychiatric patient.

Based on a sample of 174 patients released from a state hospital and followed for 6 months, a study of the predictive ability of various health professionals with respect to the financial burden and psychological burden of the patient to the family and two related aspects of his community life-family's welcome, and patient's work capacity-was performed. Although the professionals are doing a "good job" in predicting family welcome, the results are less encouraging for the other variables considered. With scattered exceptions, interdisciplinary differences were not detected.

In recent years there has been a well-documented decrease in the length of stay of psychiatric patients in inpatient facilities. There may be definite advantages in returning the psychiatric patient to the community, but one must also be concerned with the potential burdens that such patients place on the community. In order to accomplish the goal of short-term inpatient stay and to minimize the burden to the family and community, it is essential that the health professional (psychiatrist, registered nurse, attendant nurse, etc.) be able to predict accurately at time of release, the burden of the patient to the family and community.

This paper will be concerned with two specific burdens of the patient on his family-financial burden and psychological and social burden. Closely related to these, two additional aspects of the patient's adjustment to home and community life-the family's welcome of the patient and the patient's work capacity-will be investigated. The primary emphasis will be on how accurately various health professionals can predict

${ }^{\star}$ Dr. Williams is Assistant Professor in the Department of Biostatistics, School of Public Health, University of Michigan, Ann Arbor, Michigan, 48104. Dr. Hsu is Assistant Professor in the Department of Industrial Engineering, School of Engineering, Wayne State University, Detroit, Michigan. Dr. Lin is Professor of Mental Health and Professor of Psychiatry, University of Michigan, Ann Arbor, and is principal investigator of "Evaluation of Continuity of Care of Mental Patients" which is supported by a 314 (d) grant from the Michigan Department of Mental Health, Lansing, Michigan. The present study has been carried out as a subproject of the above. The authors owe sincere gratitude to the following colleagues without whose whole-hearted cooperation, the study could not have been possible: all the staff of the Genesee Division Pontiac State Hospital, especially Dr. N.F. Castido, Director, Miss Barbara Metschke, Nursing Director; and all the staff of the Genesee County Community Mental Health Agency who participated in the project, especially, Commissioner Dr. Ronald Chen. Clinical Director Mrs. Betty Curtis, Chief of Biometrics Donald Thompson, Head Nurse Lonni Huel. 
at the time of release these burdens and related factors. This study will be based on a 6-month follow-up of 174 patients released from a state hospital.

\section{REVIEW OF THE LITERATURE}

There has been a growing awareness in recent years of the multitude of considerations that are involved in the decision to release a patient from a psychiatric hospital. Lorei and Gurel (1971) have considered a decision-theoretic approach to the problem and have suggested that discussion of what is likely to happen to the patient upon release may be helpful. In fact, Lorei (1968) has postulated that treatment personnel make projections about the possible outcome of release versus retention, and that they base their decision for release on the value they place on those outcomes.

Gunn, Pearman, Goeth, Wagner, and Harrelson (1970) found that health professionals placed great importance on social and environmental factors when making a decision to release a psychiatric patient. Their definition of social factors included the patient's social functioning and his family and interpersonal relationships. The environmental factor included community acceptance and availability of employment. Gunn and Pearman (1970) also found that family relationships and community understanding were important factors in the future outlook of hospitalized psychiatric patients.

Lasky, Hover, Smith, Bostian, Duffendack, and Nord (1959) in a rare study of its kind investigated the ability of staff members to predict 2year posthospital adjustment for psychiatric patients. Their results showed that staff members predicted with $70 \%$ accuracy on the average.

Similar to certain aspects of Lasky et al. (1959), the study presented here is concerned with the ability of health professionals to predict aspects of family and community life alluded to by Gunn et al. (1970) and Gunn and Pearman (1970). However, the present study appears to be unique in its emphasis on the burden of the released mental patient on the family.

\section{STUDY DESIGN}

In our sample, all 174 patients released from the Genesee Division of Pontiac State Hospital during the 6-month period from November 1, 1970, through April 30, 1971, were studied. Data for this study were divided into two phases, each of which required the completion of a set of questionnaires. At the time of discharge, "release forms" were given to different health professionals to be completed. We will only be concerned with the release forms completed by the psychiatrist, registered nurse, attendant nurse, social worker, and occupational therapist. In most cases, the patient to be released was not known by a member of each of these professional groups. Moreover, each patient was not 
seen by the same psychiatrist, registered nurse, etc. In fact, five psychiatrists, eight registered nurses, a large number of attendant nurses, two social workers, and one occupational therapist were involved with these 174 patients. The patient's family was also required to complete separate questionnaires, primarily designed to assess the benefits of hospitalization and their views on the patient's prospects. Within 2 weeks following release, each patient was requested to make a visit to the Genesee County Community Mental Health Agency (GCCMHA) in Flint, Michigan. At this time a clinician independently interviewed and evaluated the patient's condition and future prospects. The same clinician did not interview all 174 patients. The clinicians involved in this evaluation were two psychiatrists and a large number of other staff members at GCCMHA. The second phase of the study consisted of a 6-month follow-up form completed by a clinician at the Flint Mental Health Clinic during an interview with the patient and the patient's family or someone living with him.

This prediction study on the burden of released mental patients is part of a project entitled "Evaluation of Continuity of Care of Mental Patients." Concurrently, the prediction by health professionals of the clinical course and readmission status of released mental patients is being investigated. A parallel study is also presently being conducted at the Neuropsychiatric Institute (NPI) of the University of Michigan. In this parallel study the ability of staff members to predict adjustment of psychiatric inpatients 3 months after release is being examined. The results of these and other studies will be reported on at a later time.

\section{FAMILY WELCOME}

Before considering the financial and psychological and social burden of the patient on the family, the aspect of family welcome will be considered. Due to certain simplicities that arise in presentation, this order was chosen as a means to introduce the specific type of analyses employed in the remainder of the study.

On the release forms completed by the psychiatrist, social worker, registered nurse, and occupational therapist and on the form completed by the clinician at GCCMHA, the following question was asked: "Do you think the patient's family will really welcome the patient home?" On the form completed by the patient's family, the following question was asked: "Do you welcome the patient home?" The possible answers to both questions were "No," "Yes," "I don't know," and "Not applicable." The "Not applicable" response was primarily developed for the patient who had no family or was not returning home. Cross-tabulation of the four possible responses provided by a given health professional and the four possible responses provided by the family were prepared for the six different health professions considered in this study.

Let us first consider the predictive ability of the psychiatrists with re- 
spect to family welcome. In order to avoid ambiguity, we have chosen to eliminate the "I don't know" and "Not applicable" responses that occurred either on the health professional or the family form. Thus we are left with 100 of the original 174 patients to be studied. There is a possibility that those patients for whom prediction is most difficult have been eliminated. Two statistical procedures were employed in the analysis of the question, "For the patient's considered, can the health professional predict family welcome?" First, a chi-square test for homogeneity was performed in order to compare the family responses in the group for which the psychiatrist stated the patient would be welcome to the family responses for the group for which he said they would not be welcome. The test was found to yield a significant result at $\alpha \leq .05$. (All significance levels reported were determined by use of the Boneferroni inequality for six tests corresponding to the six professions). Second, nominal $95 \%$ confidence intervals for the fraction predicted correctly, $p$, were prepared. For the psychiatrist, $.70 \leq p \leq .86$. Both procedures were used not only to evaluate how often the health professional was correct in his prediction but also to determine if the patients he categorized differently were, in fact, viewed differently by the family.

No analyses were prepared for the occupational therapist due to small sample sizes. The chi-square test for homogeneity was significant at $\alpha \leq .05$ for social workers and GCCMHA clinicians and was significant at $\alpha \leqslant .10$ for registered nurses and attendant nurses. The results of the confidence interval are summarized in Table 1 . (Because different groups of patients are involved in the analysis for the different health professions, the above analysis is not intended to be used to make comparisons between professionals. Such comparisons will be made later in the paper by a different procedure.)

From the above analysis it appears that each health profession is doing a "good job" in predicting family welcome. One should also note that although the patients the psychiatrist categorized differently were, in fact, viewed differently by the family, there is still considerable inaccuracy in prediction for those patients for whom the psychiatrist says they will not be welcome by the family. This pattern appears to be true for the other health professionals considered. Perhaps this inaccuracy is not only a function of the health professional, but also of the family who may feel certain social pressures or may for other reasons give an affirmative answer to such a question when, in fact, the patient is not welcome.

There is another question that we should examine. "Does profession $X$ do a better job than profession $Y$ in predicting family welcome?" Due to differences in training and contact with the patient, one might expect, a priori, that some professions may do a better job than others. In order to answer this question, tables were prepared for each two professions cross-classifying those patients for whom the accuracy of both professions could be determined. The number of patients so cross-classified varied, of course, depending on which particular combination of profes- 


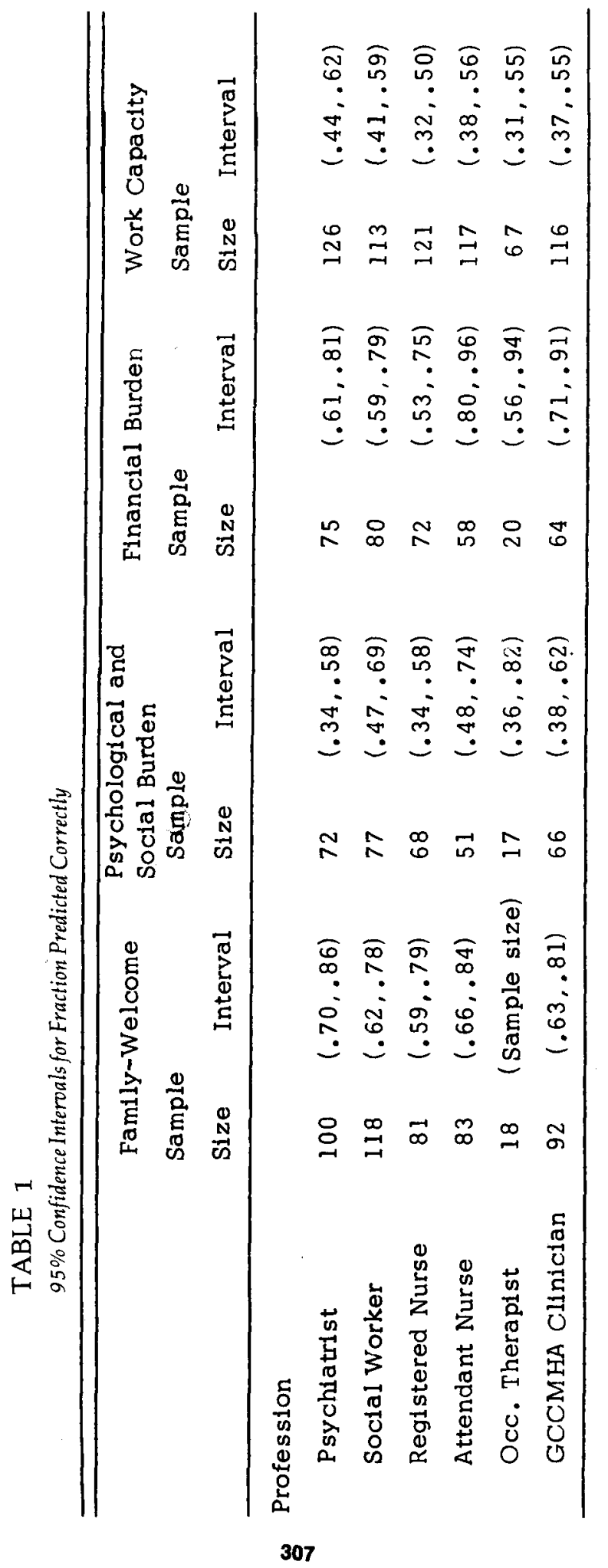


sions were examined. For the attendant nurse and occupational therapist only 14 patients were studied whereas for the psychiatrist and social worker, 87 patients were studied. By means of McNemar's test and by using the Bonferroni inequality to control the overall error rate for the 15 comparisons at $\alpha \leqslant .15$, no test gave a significant result. Therefore, we cannot conclude that any profession can predict family welcome better than any other profession.

\section{PSYCHOLOGICAL AND SOCIAL BURDEN}

On the forms completed by the various health professionals at time of release of the patient, the following question was asked: "On the basis of your observation, do you think the patient's family will be psychologically and/or socially burdened by the patient's return?" The possible answers were "No," "Yes," "I don't know," and "Not applicable." If the response was "Yes," the respondent was asked to check one or more of the following categories: (a) constantly worrying about his condition: (b) will be spending a considerable amount of time keeping company with the patient: (c) will be dreading that the patient may become sick again and return to the hospital: (d) will be worrying about the possibility of the patient creating disturbances to others: (e) will be ashamed of his being seen by their neighbors or others in the community: ( $f$ ) family will be restricted in getting together with others: (g) family will be restricted in their recreation and vacation: (h) other. These specific responses have been included here as a means of defining what is meant by a psychological or social burden.

On the 6-month follow-up form, a similar question was asked: "Is it difficult for the family to have the patient at home?" The interviewer was instructed to check as many of the following responses as needed: (a) no difficulty at all: (b) yes, because he needs help with daily life: (c) yes, because he has to be watched: (d) yes, because he limits the family's social activities: (e) yes, because he is a financial burden: ( $f$ ) yes, because the family worries about him: $(\mathrm{g})$ yes, because he upsets the family and others: (h) do not know: (i) other: (j) not applicable. Although these responses are not exactly the same as the implied definition presented on the release form, the implication of the two questions, in terms of a yes -no response, appears to be clearly the same except for the response at follow-up concerning financial burden. Therefore, for this analysis all patients who at follow-up had a response of (e) only will be eliminated. All patients with "I don't know" or "Not applicable" at release were also omitted. Moreover, those patients with "I don't know," "Not applicable," or "Other" at follow-up were omitted. Finally, cross-tabulations of a yes or no response at release and a yes or no at follow-up (where yes was defined as any combination of the responses (b), (c), (d), (f), or (g); or any combination of the responses (b), (c), (d), (f), or (g) in combination with (e) were prepared for each profession. 
As before, the predictive ability of psychiatrists with respect to psychological and social burden will be considered first. After the elimination of patients for whom no clear determination of the accuracy of prediction could be made, 72 of the original 174 patients are left to be studied. Again a chi-square test for homogeneity was performed. The test was not significant at any reasonable level of significance. A nominal $95 \%$ confidence interval for $p$ was prepared; for the psychiatrist, $.34 \leq p$ $\leq .58$.

The chi-square test for homogeneity was not significant at any reasonable level of significance for social workers, registered nurses, attendant nurses, and GCCMHA clinicians. No test was performed for the occupational therapist due to small sample sizes. The results of the confidence intervals are summarized in Table 1 . From the above analysis, one cannot conclude that any health profession is doing a "good job" in predicting psychological and social burden.

Although we cannot conclude that any health profession is doing a good job, it still may be possible that one profession is doing better than another. Employing the same method as described earlier to investigate this question, we cannot conclude that any one profession can predict psychological or social burden better than any other profession. Again, the number of patients studied in each comparison varies. In this case the number of patients considered ranges from 11 for the attendant nurse and occupational therapist comparison to 63 for the psychiatrist and social worker comparison.

\section{FINANCIAL BURDEN}

On the forms completed by the various health professionals at time of release of the patient, the following question was asked: "Do you think the patient may be a financial burden on the family?" The possible answers were "No," "Yes," "I don't know," and "Not applicable." On the 6-month follow-up form a similar question was asked: "How does the family feel about the patient's financial effect?" The interviewer was instructed to check only one of the following responses; (a) the family is not affected financially: (b) the family accepts it, it is not a burden: (c) it is a slight burden: (d) it is a great burden: (e) it varies: ( $f$ ) other: ( $g$ ) do not know: (h) not applicable. Responses (b) and (e) in the follow-up imply a slightly different interpretation than the question asked at release. In order to avoid the ambiguities that could arise by including these patients in our analysis, patients with a response of (b) or (e) at follow-up were eliminated along with those patients who had an "I don't know" or "Not applicable" response at release or an "Other," "I don't know," or "Not applicable" response at follow-up. Because the question asked at release did not allow for gradations of burden, the responses of (c) and (d) at follow-up were combined. Finally, cross-tabula- 
tions of a yes or no response at release and a yes or no response at follow-up were prepared for each profession.

The predictive ability of psychiatrists with respect to financial burden will be considered first. After patients for whom no clear determination of the accuracy of prediction could be made were eliminated, 75 of the original 174 patients are left to be studied. A chi-square test for homogeneity was performed and found to be not significant at any reasonable level of significance. A nominal 95\% confidence interval for $p$ was prepared; for the psychiatrist, $.61 \leq p \leq .81$. In order to interpret these statements, one should refer to Table 2 . From Table 2 it is clear that at follow-up $77 \%$ of the patients studied caused no financial burden. Thus the psychiatrist could appear from a confidence interval statement for $p$ to be doing a "good job" had he simply said that no patient would be a financial burden. Clearly, however, we would not want to say that the the psychiatrist is doing a "good job" under this latter approach. Therefore, because the confidence interval approach in this case appears to be misleading, conclusions will be based primarily on the test for homogeneity. Thus psychiatrists can predict fairly accurately those patients who are not a financial burden but cannot predict those patients who are a financial burden.

The chi-square test for homogeneity was not significant at any reasonable level of significance for social workers and registered nurses. No such test was performed for attendant nurses, occupational therapists, and GCCMHA clinicians due to small sample sizes. The results of the confidence intervals are summarized in Table 1. From the above analysis, it appears that we cannot conclude that any health profession is doing a "good job" in predicting financial burden for both those patients who are a burden and those patients who are not a burden.

However, although we cannot conclude that any health profession is doing a good job of prediction, it would be of interest to identify those professionals who are doing the best job of prediction in order to develop an understanding of how health professionals, in general, could improve their prediction of financial burden. By means of McNemar's test we can

TABLE 2

Financial Burden-Psychiatrist

\begin{tabular}{cccc}
\hline & Follow-up & & \\
& No & Yes & Total \\
\hline Release & & & \\
No & 45 & 9 & 54 \\
Yes & 13 & 8 & 21 \\
Total & 58 & 17 & 75 \\
\hline
\end{tabular}


TABLE 3

Financial Burden

\begin{tabular}{cccc}
\hline & $\begin{array}{l}\text { Attendant Nurse } \\
\text { Correct }\end{array}$ & Incorrect & Total \\
\hline Soclal Worker & 38 & 0 & 38 \\
Correct & 10 & 4 & 14 \\
Incorrect & 48 & 4 & 52 \\
Total & & & \\
\hline
\end{tabular}

conclude that the attendant nurse is doing a better job than the social worker and a better job than the registered nurse (see Tables 3 and 4). No other comparisons were significant. The number of patients considered in each comparison varied from 12 for the occupational therapist and therapist comparison to 67 for the psychiatrist and social worker comparison.

\section{WORK CAPACITY}

On the forms completed by the various health professionals at time of release of the patient, the following question was asked: "What is your view regarding the patient's ability to work?" The possible answers were "Full capacity," "Half capacity," "Minimal capacity," "Not able to work," "I don't know," and "Not applicable." On the 6-month follow-up form a series of questions related to this question were asked. These questions are listed below along with their possible answers.

1. Is the patient working for pay now? Circle one.

Answer: (a) No, not working; (b) Yes, working as an employer; (c) Yes, working as an employee; (d) Yes, working in a sheltered workshop; (e) Has worked for pay in last 6 months, but is not now; (f) Other; (g) I don't know; (h) Not applicable.

\section{TABLE 4}

Financial Burden

\begin{tabular}{clcc}
\hline & $\begin{array}{l}\text { Attendant Nurse } \\
\text { Correct }\end{array}$ & Incorrect & Total \\
\hline Registered Nurse & & & \\
Correct & 26 & 1 & 27 \\
Incorrect & 13 & 4 & 17 \\
Total & 39 & 5 & 44 \\
\hline
\end{tabular}


2. If working now for pay, please answer the following question: Does the patient work full time?

Answer: (a) Yes, full time; (b) Part time; (c) It varies; (d) Other; (e) I don't know; (f) Not applicable.

3. Does the patient do work at home or around the house?

Answer: (a) Not at all; (b) Head housekeeper; (c) Helps others with housekeeping; (d) Works as unpaid family worker; (e) Other; ( $f$ ) I don't know; (g) Not applicable.

4. If patient does any work at home, please answer the following question. Is this full time work?

Answer: (a) Yes; (b) Part time; (c) It varies; (d) Other; (e) I don't know; (f) Not applicable.

5. If the patient has attended school or classes, please answer the following question. Is the patient enrolled in these classes full time?

Answer (a) Yes; (b) Part time; (c) It varies; (d) Other; (e) I don't know; (f) Not applicable

Based on these five questions it was possible to classify a patient as to whether he was demonstrating a clear capacity to work, minimal capacity, no capacity, or unknown capacity. Without stating the specifics of the way in which such a decision was made, a brief description of what is meant by those categories at follow-up will be given. A person with a clear capacity to work will (1) be working at least part time as an employer or employee, (2) be working at least part time as a head housekeeper or unpaid family worker, or (3) be at least a part-time student. A person with minimal capacity to work will not have demonstrated a clear capacity but will at least (1) be working in a sheltered workshop, (2) have worked for pay in the last 6 months, (3) be working a variable amount as an employer, employee, or unpaid family worker, or (4) be spending a variable amount of time as a student. Someone with no capacity to work will (1) not be working for pay now, (2) be doing no work at home, and (3) not be a student.

In order to make the responses at release and follow-up more comparable, the responses of full capacity and half capacity at release were combined. Those patients with a response of "I don't know" or "Not applicable" at release or unknown at follow-up were omitted. Thus crosstabulations of responses of clear, minimal, or no capacity at release and responses of clear, minimal, or no capacity at follow-up were prepared for each professional.

Admittedly it is possible that a released mental patient may "inherently" have a clear capacity to work but for various reasons not be able to demonstrate that ability according to the criteria described above. It is felt that only a few such cases are present in this sample and will not cause any significant changes in the conclusions made.

The predictive ability of psychiatrists with respect to work capacity will be considered first. After those patients for whom no determination of the accuracy of prediction could be made were eliminated, 126 of the original 174 patients were left to be studied. A chi-square test for homogeneity was performed and found to be a significant at $\alpha \leq .05$. A nomi- 
nal $95 \%$ confidence interval for $p$ was prepared; for psychiatrist, $.44 \leq p \leq .62$. From Table 5 it is seen that the psychiatrist made quite a few errors in prediction, but at least most of those patients for whom he predicted a clear capacity to work demonstrated that capacity; those patients for whom he predicted minimal capacity are classified about equally in all three follow-up groups; and most of those patients for whom he predicted no capacity are not demonstrating any capacity.

The chi-square test for homogeneity was significant at $\alpha \leq .05$ for social workers. The test for homogeneity was not significant at any reasonable level of significance for GCCMHA clinicians. No such test was performed for registered nurses, attendant nurses, and occupational therapists due to small sample sizes. The results of the confidence intervals are summarized in Table 1 . From the above analyses we cannot conclude that any health professional is doing a good job in predicting work capacity. However, there is an indication that psychiatrists and social workers have some understanding of this capacity in their patients.

By means of McNemar's test we can conclude (at $\alpha \leqslant .15$ ) that psychiatrists do a better job than registered nurses in predicting work capacity (see Table 6). No other comparisons were significant. The number of patients considered in each comparison varied from 58 for the attendant nurse and occupational therapist comparison to 118 for the psychiatrist and registered nurse comparison.

\section{DISCUSSION}

Based on a sample of 174 patients released from a state mental hospital and followed for 6 months, a study of the predictive ability of various health professionals with respect to the financial burden and psychosocial burden of the patient to the family and two related aspects of his community life-family's welcome and his work capacity-was performed. In the study only those patients for whom a clear determination of the accuracy of the prediction could be made were included. We cannot conclude that any profession is doing a "good job" in

TABLE 5

Work Capacity-Psychịatrist

\begin{tabular}{|c|c|c|c|c|}
\hline \multicolumn{5}{|c|}{ Follow-up } \\
\hline & Clear & Minimal & None & Total \\
\hline \multicolumn{5}{|l|}{ Release } \\
\hline Clear & 47 & 10 & 23 & 80 \\
\hline Mintmal & 7 & 4 & 13 & 24 \\
\hline None & 5 & 1 & 16 & 22 \\
\hline Total & 59 & 15 & 52 & 126 \\
\hline
\end{tabular}


predicting psychological and social burden. Moreover, no one profession is doing a better or worse job than another in this prediction. For financial burden it appears that health professionals may be able to predict fairly accurately those patients who are not a financial burden but cannot predict those patients who are a financial burden. There is evidence that the attendant nurse seems to be doing a better job in predicting financial burden than social workers or registered nurses, but no other comparisons between professionals show any significant differences. For work capacity there is no indication that any profession is doing a "good job" in prediction. However, there is some indication that psychiatrists and social workers have some understanding of the situation.

In contrast, it appears that all of the health professionals considered are doing an equally "good job" in predicting family's welcome. This may be due to the fact that the attitude of the family to the patient is more readily perceived by the staff in their routine clinical work and, furthermore, the usual emphasis on observing the patient-family relationship is likely to direct the attention of the clinical staff to this particular aspect of the patient's life. Conversely, the understanding of the financial or psychological burden of the patient to his family requires specific enquiry that does not seem to form part of the routine work of the staff with the mental patients. This is unfortunate, as the ability to foresee the possible financial and psychosocial burdens to the family and the patient's work capacity in advance of his release would have an important effect upon the decision to release the patient as well as on the management of the patient's care after release.

No significant difference was noted between the state hospital staff and the GCCMHA staff in their ability to predict the factors under consideration. This result is contrary to the expectation that the former may perform better by virtue of their longer and more intimate contact.

The fact that the data presented here did not detect interdisciplinary differences, with scattered exception, in the ability to predict the four factors under consideration deserves special mention. By virtue of specific training background and responsibility, we would expect a certain pro-

TABLE 6

Work Capacity

Registered Nurse

Correct

Incorrect

Total

Psychiatrist

Correct

Incorrect 
fession would do better than the other(s) in foreseeing certain aspects of patient's posthospital life: for example, social workers would do a good job in predicting the financial burden of the patient to his family or the registered nurse in predicting the psychosocial burden of the patient to the family, etc.

Since in this study many of the statistical tests were nonsignificant, one should always be concerned about the power (related among other things to sample size) of those statistical tests when making interpretations. Perhaps future studies in which there is no need to eliminate some patients from statistical analysis due to ambiguous responses and in which a larger sample of patients is available would help clarify some of the issues raised during the present study.

A further question may be raised, "How well are the family members able to foresee the possible impact on themselves of the patient's return home?" The data bearing on this question will be presented in another paper.

\section{REFERENCES}

Gunn, R.L. \& Pearman, H.E. An analysis of the future outlook of hospitalized psychiatric patients. Journal of Clinical Psychology, 1970, 26, 99-103.

Gunn, R.L., Pearman, H.E., Goeth, C., Wagner, M.K., \& Harrelson, W. Facts influencing release decision. Hospital and Community Psychiatry, 1970, 21, 290-293.

Lasky, J.J., Hover, G.L., Smith, P.A., Bostian, D.W., Duffendack, S.C., \& Nord, C.L. Posthospital adjustment as predicted by psychiatric patients and by their staff. Journal of Consulting Psychology, 1959, 23, 213-218.

Lorei, T.W. Release decision-making. (Project 60), Washington, D.C.: Program Evaluation Staff, Veterans Administration Hospital, 1968.

Lorei, T.W., \& Gurel, L. A systematic approach to disposition decision. American Journal of Psychiatry, 1971, 128, 61-65.

(The authors wish to thank Dr. Gail Barton, Department of Psychiatry, University of Michigan for pointing out the article by Lasky, et al. 1959.) 